\title{
Real Time Selective Harmonic Minimization for Multilevel Inverters Connected to Solar Panels Using Artificial Neural Network Angle Generation
}

\author{
Faete Filho, Leon M. Tolbert, Yue Cao \\ Electrical Engineering and Computer Science \\ The University of Tennessee \\ Knoxville, Tennessee USA \\ ffilho@utk.edu, tolbert@utk.edu, ycao6@utk.edu
}

\author{
Burak Ozpineci \\ Oak Ridge National Laboratory \\ Oak Ridge, Tennessee USA \\ ozpinecib@ornl.gov
}

\begin{abstract}
This work approximates the selective harmonic elimination problem using Artificial Neural Networks (ANN) to generate the switching angles in an 11-level full bridge cascade inverter powered by five varying DC input sources. Five $195 \mathrm{~W}$ solar panels were used as the DC source for each full bridge. The angles were chosen such that the fundamental was kept constant and the low order harmonics were minimized or eliminated. A non-deterministic method is used to solve the system for the angles and to obtain the data set for the ANN training. The method also provides a set of acceptable solutions in the space where solutions do not exist by analytical methods. The trained ANN shows to be a suitable tool that brings a small generalization effect on the angles' precision.
\end{abstract}

\section{INTRODUCTION}

A number of technical papers using Selective Harmonic Elimination (SHE) or minimization have been reported for fundamental frequency operation using the most common multilevel (ML) inverter topologies [1-3]. The cascade multilevel configuration has independent DC sources that may have different voltage levels. Those DC sources might be capacitors, fuel cells or solar panels and will consequently bring a voltage unbalance depending on the system dynamics. Numerous papers have been reported using selective harmonic elimination or minimization of cascaded multilevel inverters.

In [4], genetic algorithms have been used to determine the optimal switching angles for DC sources of equal values. Analytical solutions for this problem using the theory of symmetric polynomials were also reported for unipolar and bipolar schemes [5-6]. All of these papers assumed that the DC sources are equal and do not vary with time. In [7] and [8], analytical solutions for the case of unequal DC sources have been derived and in [9-10] algorithms to solve for the angles have been proposed. Also, in [11-12] a more general approach is formulated for the $m$-level $n$-harmonic case. All of these papers use computationally intensive time consuming equations to solve for the angles; therefore, the switching angles are calculated off-line.

The authors of [13-14] have developed methods to calculate the switching angles in real-time; however, their approach was not extended for unequal DC sources. An alternate approach to determining the optimum switching angles in real-time for varying DC sources is to calculate the switching angle solutions off-line and store the solutions in a look-up table. For accurate representation of every solution for every different DC source case, a huge look-up table would be required. Even then, for some operating points, the solutions might be missing and some type of interpolation would be required.

In this paper, the look up table is replaced by an Artificial Neural Network (ANN), which if well trained, has the inherent capability of generalizing solutions. What this means is that, if the correct range of data are used for training and if the ANN is not over-trained, the network will fill in the solution gaps properly. Since ANN runs fast, it is possible to quickly determine the switching angles to establish real-time control.

\section{APPROACH TO THE Problem AND Methodology}

A system's overview of the 11-level cascade inverter and control is presented in Fig. 1. It has a five full bridge series connected configuration with five solar panels as its isolated input DC supply that may have different voltage levels and/or dynamics if the photovoltaic modules connected to each Hbridge have different characteristics.

\section{A. Selective Harmonic Elimination and Unequal DC Sources}

Equation (1) shows the contents of the output voltage at infinite frequencies. The module voltages $V_{p v l}$ to $V_{p v 5}$ are associated to their respective switching angles $\theta_{1}$ to $\theta_{5}$. This equation includes only odd, non-triplen harmonics. The reason for that lies on the assumptions of wave symmetry that cancels out the even components and also in a three phase application the multiples of the third harmonic vectors will add up to zero in the line voltage of a three phase balanced system. Equation (1) is the core equation and also starting point for SHE, because the decision for the target harmonics will define the set of transcendental equations to be solved. It is desired to solve (1) so that under variations in the DC input sources the fundamental output voltage is maintained and the lowest nontriplen harmonics, in this case the 5th, 7th, 11th and 13th are canceled. In applications for a three phase machine drive, there is no need to cancel the harmonics that are a multiple of three because these are cancelled in the line voltage. 

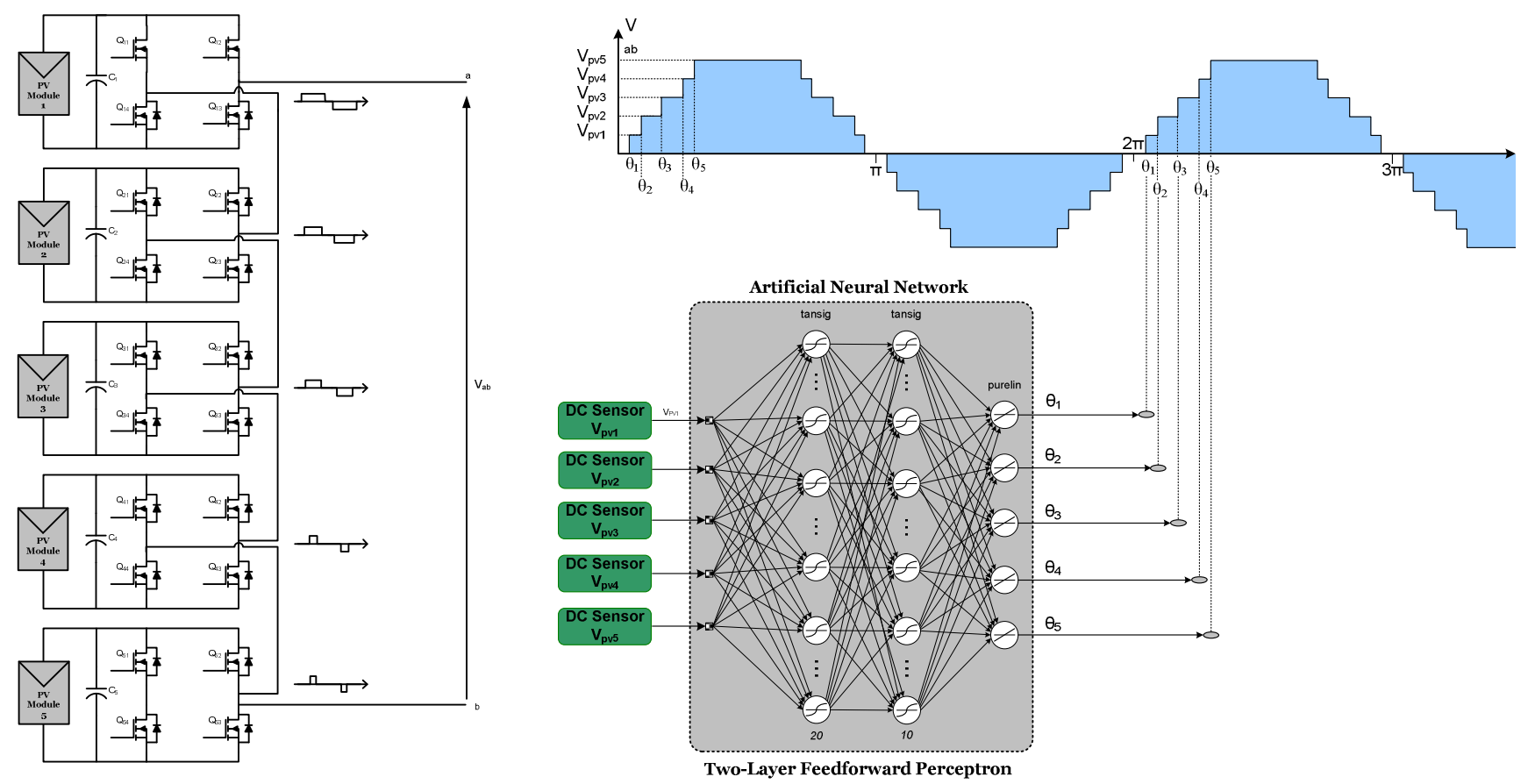

Fig. 1. Multilevel cascade inverter topology and ANN-based angle control.

$$
V_{a b}(w t)=\sum_{n=1,5,7,11,13}^{\infty} \frac{4}{\pi \cdot n} \cdot\left(V_{P V 1} \cos \left(n \cdot \theta_{1}\right)+V_{P V 2} \cos \left(n \cdot \theta_{2}\right)+V_{P V 3} \cos \left(n \cdot \theta_{3}\right)+V_{P V 4} \cos \left(n \cdot \theta_{4}\right)+V_{P V 5} \cos \left(n \cdot \theta_{5}\right)\right.
$$

Many DC sources such as solar panels and fuel cells have varying output voltages depending on varying sunlight intensity, load, or other factors. For grid connection, either a DC-DC converter is used to regulate this DC voltage, or the modulation index of the grid-interface inverter is varied depending on the DC voltage level. For example, during a day of operation, the solar panel output voltage may vary according to the amount of energy available, and the grid interface system should be able to respond to this variation in the switching angles to keep the fundamental regulated at its reference value and the low order harmonics minimized.

The approach in this work is to maintain the fundamental at the desired level by means of choosing the low frequency switching angles in (1) as shown in Fig. 1. Instead of using an analytic method to determine the angles off-line, this paper uses a non-deterministic approach to solve for the angles as it can give some insight about the convergence of the solution in the space where no solution can be found by the analytical approach. In this manner, a Genetic Algorithm (GA) was implemented to find the switching angles (off-line) for a set of pre-determined input voltages of an 11-level cascade inverter. An important feature of the GA for this approach is that for the range space where there is no analytical solution, the GA will find the nearest solution providing a smooth data set that is needed for the ANN training. Then, with the previous data set, the ANN was trained to output the set of angles for each input voltage situation.

\section{B. Solar Cell Modeling}

A suitable model was derived to simulate the PV module behavior that reflects the experimental curves of the solar panel with relative accuracy. The single diode model was adopted as shown in Fig. 2 to simulate the PV module under different irradiance and temperature levels. A number of approaches and models can be found in the literature to analyze the behavior of PVs that can grow in complexity if even better accuracy is needed [15-17]. The suitable model becomes then application dependent.

The PV cell model used in this work is a more intuitive model based on the single diode cell (Fig. 2) and derived in

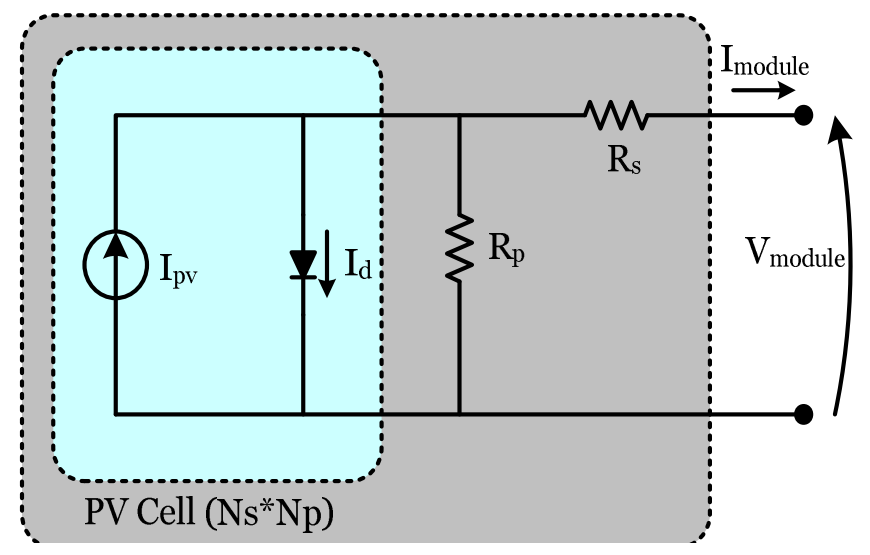

Fig. 2. Single diode model representation of a photovoltaic module. 


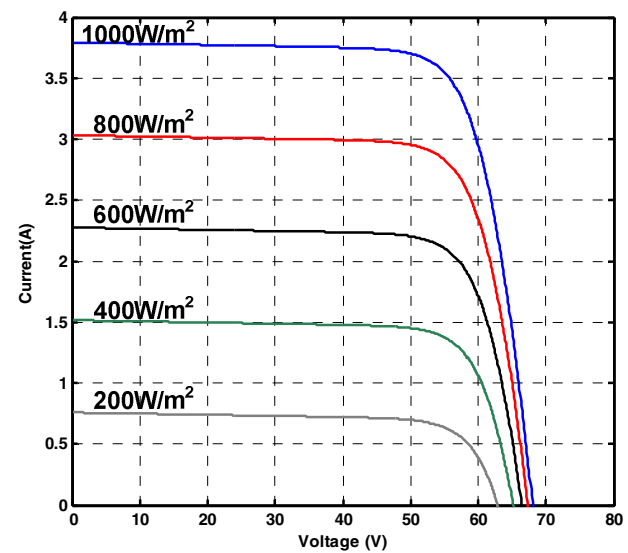

(a)

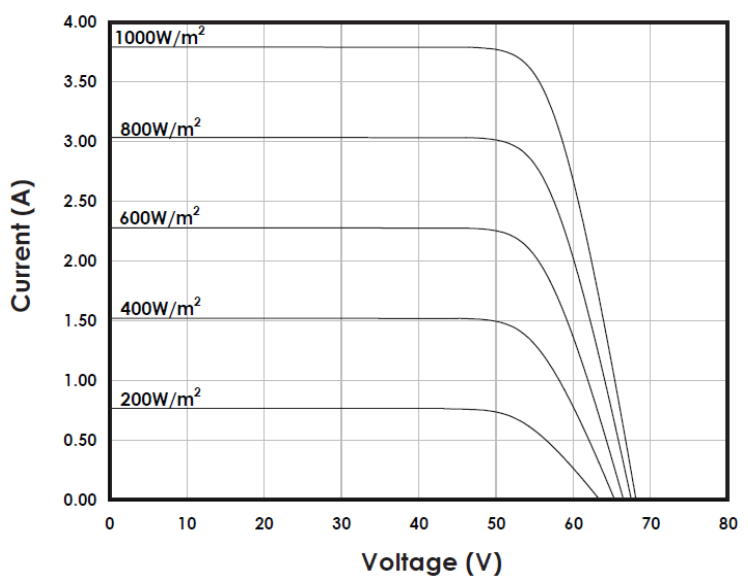

(b)

Fig. 3. (a) Simulated and (b) experimental I-V curves for Sanyo HIT 195 photovoltaic module.

[18] where the inputs used are those obtained directly from the PV module datasheet parameters which are readily available from a panel's manufacturer. This model greatly simplified the modeling task once the iterations and non linear equations were solved. Equation (2) is the basic equation and the Solar Panel's datasheet provides the equation values to solve for the unknowns.

$$
I=I_{p v}-I_{0} e^{\frac{V+R_{S} I}{V_{t} a}}-1-\frac{V+R_{S} I}{R_{p}}
$$

where,

$I_{p v}$ : photovoltaic current

$I_{0}$ : saturation current

$V_{t}$ : thermal voltage

$R_{s}$ : equivalent series resistance

$R_{p}$ : equivalent parallel resistance

$a$ : diode ideality constant

The solar panel to be connected to the H-bridge inverter is a Sanyo HIT 195W and its specifications and simulated parameters for use with (2) are shown in Table 1 and 2, respectively.

TABLE I. SIMULATED PARAMETERS FOR EQUATION 2

\begin{tabular}{|c|c|}
\hline Parameter & Value \\
\hline$I_{p v}$ & $3.794 \mathrm{~A}$ \\
\hline$I_{0}$ & $9.68 \times 10^{-10} \mathrm{~A}$ \\
\hline$V_{t}$ & $2.466 \mathrm{~V}$ \\
\hline$a$ & 1.25 \\
\hline$R_{s}$ & $1.0973 \Omega$ \\
\hline$R_{p}$ & $1060.0 \Omega$ \\
\hline
\end{tabular}

TABle II. SANyo HIT PV Module Electrical SPeCifications

\begin{tabular}{|l|c|}
\hline Model & HIP-195BA19 \\
\hline Rated Power (Pmax) & $195 \mathrm{~W}$ \\
\hline Max. Power Voltage (Vpm) & $55.3 \mathrm{~V}$ \\
\hline Max. Power Current (Ipm) & $3.53 \mathrm{~A}$ \\
\hline Open Circuit Voltage (Voc) & $68.1 \mathrm{~V}$ \\
\hline Short Circuit Current (Isc) & $3.79 \mathrm{~A}$ \\
\hline Temperature Coefficient (Voc) & $-0.17 \mathrm{~V} /{ }^{\circ} \mathrm{C}$ \\
\hline Temperature Coefficient (Isc) & $0.87 \mathrm{~mA} /{ }^{\circ} \mathrm{C}$ \\
\hline
\end{tabular}

Fig. 3 compares the model obtained from the PV datasheet with the manufacturer's experimental curve to show the performance of the model. The power versus voltage curve also matches the manufacturer's experimental data at the same level of accuracy as shown in Fig. 3.

\section{Artificial Neural Networks}

Artificial neural networks have found a number of applications in engineering such as pattern recognition, control and classification, among others [18-21]. One of the main factors for choosing this technique is its generalization ability in nonlinear problems that are in nature complex and/or requires time consuming calculations.

The number of possible combination that generates the data set for ANN training is exponentially increased by the number of $\mathrm{H}$ bridges in the topology. For a two full bridge case (5 levels) considering a dataset of four voltage levels for training, for example, $[45 \mathrm{~V}, 50 \mathrm{~V}, 55 \mathrm{~V}, 60 \mathrm{~V}]$ would generate a table of $4^{2}$ rows. In a five $\mathrm{H}$-bridge converter with 10 points equally spaced between $50 \mathrm{~V}$ and $60 \mathrm{~V}$ it would generate $10^{5}$ different combinations. In an effort to reduce the size of the dataset, the problem is faced as a permutation problem instead of a combination. In this way, the data set can be greatly reduced by considering, for example, the voltage vector $v_{1}=(45,45,50,55,50)$ as being the same input as $v_{2}=(45,50,45,50,55)$. 


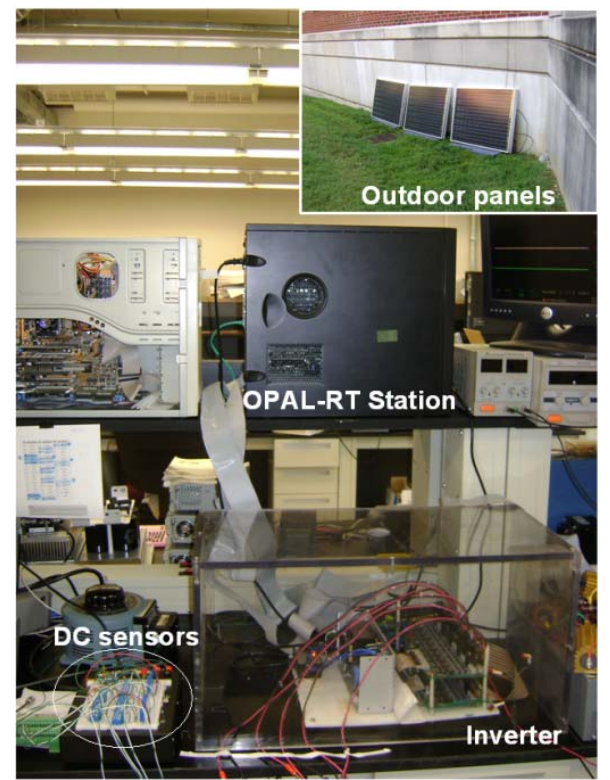

Fig. 4. Experimental multilevel setup and photovoltaic panels installation.

The ANN implemented and shown in detail in Fig. 1 is a feedforward multilayer perceptron with two hidden layers of 20 and 10 neurons that are interconnected through weighting functions. This configuration was chosen over diverse designs due to better performance in terms of training time, memorization, and learning ability.

\section{EXPERIMENTAL RESULTS}

The implemented system is pictured in Fig. 4. Each full bridge in the cascade topology has a $1 \mathrm{mF}$ capacitor and four 200V/40A MOSFETs. The OPAL-RT Lab is a hardware-inthe-loop (HIL) system that allows real time control in a network of computers. The topology used in this experiment uses two computers; one is the main station where Simulink ${ }^{\circledR}$ is installed and the control is implemented (master station); the second computer has the analog and digital I/Os to control the inverter and acquire signals (slave station). Compared to DSPs, this system has a shorter implementation and debugging time as a result of a user-friendly interface;

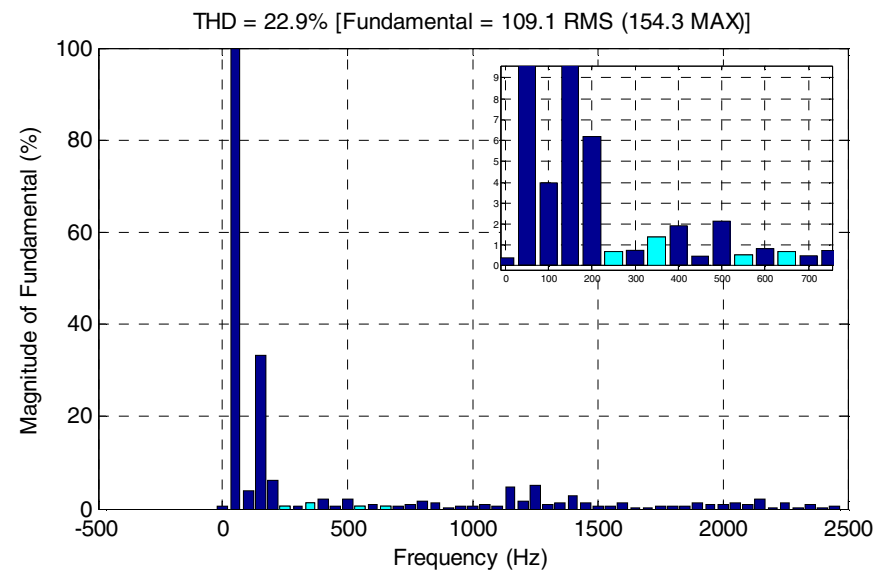

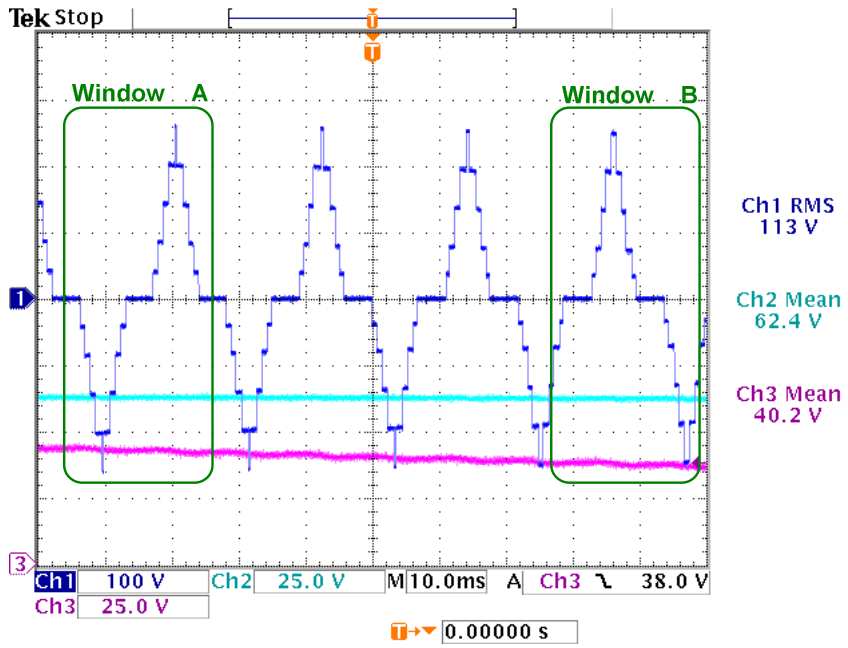

Fig. 5. Real time change in angle generation (blue) by the ANN after voltage variation (purple) over a $100 \mathrm{~ms}$ window.

however, this comes at the cost of a sample time that can range from 50 us to 150 us. This can reflect in up to one degree divergence from the software-calculated ANN angles in some cases.

Fig. 5 illustrates a $100 \mathrm{~ms}$ window of the 11-level cascade inverter running with solar panels as its input source. During this time period, a load step was applied to the inverter output causing the panels' voltage to drop. This voltage drop can be observed in channel 3 (purple) that is an indoor solar panel. This figure illustrates a resistive step change applied at the output that causes a slow voltage drop also helped by the energy stored in the capacitance. In the second channel (cyan), which is sensing an outdoor solar panel, the effect of the load step was not heavy enough to cause significant voltage drop mainly because those panels, at the time of the experiment, were subjected to a larger level of irradiance as a consequence of its orientation and time of the day.

In the same figure in the third channel (purple) a voltage drop can be seen as a result of the load change. From an initial value of $43.3 \mathrm{~V}_{\mathrm{dc}}$ the solar panel output voltage drops smoothly, also helped by the $1000 \mathrm{uF}$ electrolytic capacitor, to a value of around $36.2 \mathrm{~V}_{\mathrm{dc}}$ at the end of the acquired $100 \mathrm{~ms}$

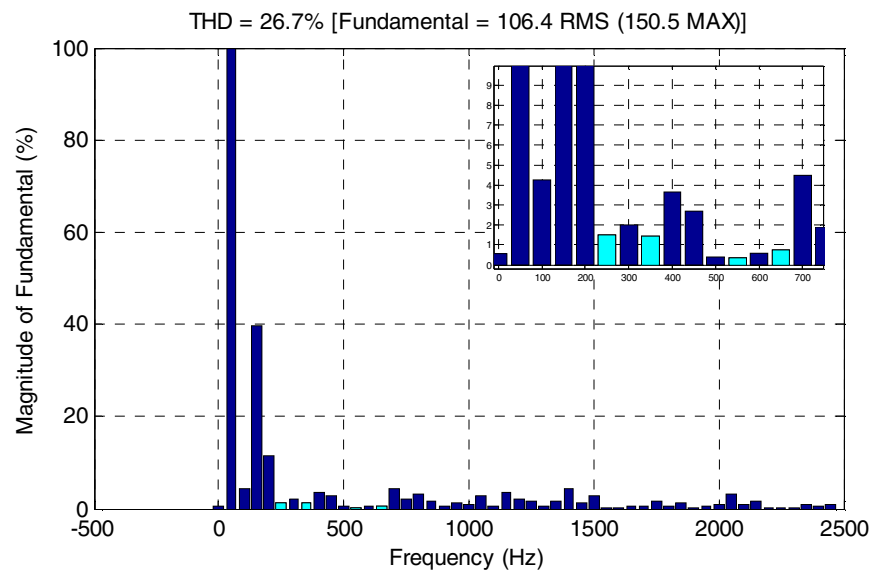

Fig. 6. Frequency spectrum content for windows A and B, respectively, as a percentage of the fundamental value. 
window. As the voltage level decreases, a new set of switching angles are calculated by the ANN to be updated at the next fundamental cycle $(50 \mathrm{~Hz}$ or $60 \mathrm{~Hz})$. The two windows shown in this figure (A and $\mathrm{B}$ ) were used to do a frequency spectrum analysis of the waveform. The change in the angles can be noticed when the two windows are compared (A and B).

The spectrum content of both windows (A and B) is shown in Fig. 6. The presence of DC and even harmonic components happen as a result of the varying voltage. It is considered in this work that such large voltage variation rate is not likely to happen, and if it occurs, it is a transitory state where the angles are updated at the cost of even and/or DC transient components. The Total Harmonic Distortion (THD) is greater than the expected range of $7 \%-14 \%$ as a consequence of those components added up by the voltage transient. The target harmonics were kept at around $1 \%$ of the fundamental as it can be seen in both windows. A zoomed window is shown in the upper right corner with the $5^{\text {th }}, 7^{\text {th }}, 11^{\text {th }}$ and $13^{\text {th }}$ components (cyan). The expected target harmonic levels obtained by the experiment are higher due to a considerably high hardware sample time during execution. The second highest component in the plots is the third harmonic, that is not considered a harmful component for three phase applications.

\section{CONCLUSIONS}

The analytic calculation of the angles in real time for harmonic elimination is still being studied and approached by researchers through different paths. This work investigated ANNs as a tool to provide the angles in-cycle $(50 \mathrm{~Hz}$ or $60 \mathrm{~Hz})$ that also can have DSP implementation but at the cost of minimization of harmonics instead of elimination. The ANN was trained with a mixed data set that has eliminated harmonics and, for those points where a solution does not exist, minimized harmonics. The real time performance over a $100 \mathrm{~ms}$ window was shown to validate the proposed approach.

The output angles returned by the ANN may not provide a satisfactory result, or harmonic elimination, at some points as it generalizes; however, a fast result can be obtained and more angles can be easily added to provide a better output waveform. Parallel networks can be used to accomplish better performance also.

Future works point in the direction of implementation and performance evaluation on a DSP platform as well as system performance over a greater number of angles with more than one ANN.

\section{REFERENCES}

[1] J. Rodriguez, J. Lai, F. Z. Peng, "Multilevel inverters: a survey of topologies, control and applications," IEEE Transactions on Industrial Electronics, vol. 49, no.4, pp. 724-738, Aug. 2002.

[2] A. Pandey, B. Singh, B. N. Singh, A. Chandra, K. Al-Haddad, D. P. Kothari, "A review of multilevel power converters," Institute of Engineers Journal (India) , pp. 220-231, vol. 86, March 2006.
[3] J. R. Wells, P. L. Chapman, P. T. Krein, "Generalization of selective harmonic control/elimination," IEEE Power Electronics Specialists Conference, pp. 1358-1363, June 2005.

[4] B. Ozpineci, L. M. Tolbert, J. N. Chiasson, "Harmonic optimization of multilevel converters using Genetic Algorithms," IEEE Power Electronics Letters, vol. 3, no. 3, pp. 92-95, Sept. 2005.

[5] J. N. Chiasson, L. M. Tolbert, K. J. McKenzie, Z. Du, "A unified approach to solving the harmonic elimination equations in multilevel converters," IEEE Trans. Power Electronics, vol. 19, no. 2, pp. 478490, Mar. 2004

[6] J. N. Chiasson, L. M. Tolbert, K. J. McKenzie, Z. Du, "Elimination of harmonics in a multilevel converter using the theory of symmetric polynomials and resultants," IEEE Transactions on Control Systems Technology, vol. 13, no. 2, pp. 216-223, March 2005.

[7] Z. Du, L. M. Tolbert, J. N. Chiasson, H. Li, "Low switching frequency active harmonic elimination in multilevel converters with unequal DC voltages," Annual Meeting of the IEEE Industry Applications Society, 2-6 Oct. 2005, pp. 92-98.

[8] Z. Du, L. M. Tolbert, J. N. Chiasson, "Active harmonic elimination for multilevel converters," IEEE Transactions on Power Electronics, vol. 21, no. 2, pp. 459-469, March 2006.

[9] M. G. H. Aghdam, S. H. Fathi, G. B. Gharehpetian, "Elimination of harmonics in a multi-level inverter with unequal DC sources using the homotopy algorithm," IEEE International Symposium on Industrial Electronics, June 2007, pp. 578-583.

[10] T. Tang, J. Han, X. Tan, "Selective harmonic elimination for a cascade multilevel inverter," IEEE International Symposium on Industrial Electronics, July 2006, pp.977-981.

[11] J. R. Wells, B. M. Nee, P. L. Chapman, "Selective harmonic control: a general problem formulation and selected solutions," IEEE Transactions on Power Electronics, vol. 20, no. 6, pp. 1337-1345, Nov. 2005.

[12] M. S. A. Dahidah, V. G. Agelidis, "Selective harmonic elimination PWM control for cascaded multilevel voltage source converters: A generalized formula," IEEE Transactions on Power Electronics, vol. 23, no. 4, pp. 1620-1630, July 2008.

[13] D. W. Kang, H. C. Kim, T. J. Kim, D. S. Hyun, "A simple method for acquiring the conducting angle in a multilevel cascaded inverter using step pulse waves," IEE Proceedings on Electric Power Applications, vol. 152, pp. 103-111, 2005.

[14] Y. Liu, H. Hong, A. Q. Huang, "Real-time calculation of switching angles minimizing THD for multilevel inverters with step modulation," IEEE Trans. Industrial Electronics, vol. 56, no. 2, pp. 285-293, Feb. 2009.

[15] U. Boke, "A simple model of photovoltaic module electric characteristics," European Conference on Power Electronics and Applications, Sept. 2007, pp.1-8,

[16] O. Gil-Arias, E. I. Ortiz-Rivera, "A general purpose tool for simulating the behavior of PV solar cells, modules and arrays," 11th Workshop on Control and Modeling for Power Electronics, Aug. 2008, pp. 1-5.

[17] R. Ramaprabha, B. L. Mathur, "MATLAB based modelling to study the influence of shading on series connected SPVA," 2nd International Conference on Emerging Trends in Engineering and Technology, Dec. 2009, pp. 30-34.

[18] J. J. Hopfield, "Artificial neural networks," IEEE Circuits and Devices Magazine, vol. 4, no. 5, pp. 3-10, Sep. 1988.

[19] R. Aggarwal, Y. Song, "Artificial neural networks in power systems II: types of artificial neural networks," Power Engineering Journal, vol. 12, no. 1, pp. 41-47, Feb. 1998.

[20] R. Aggarwal, Y. Song, "Artificial neural networks in power systems I: general introduction to neural computing," Power Engineering Journal, vol. 11, no. 3, pp.129-134, Jun. 1997.

[21] M. J. Willis, C. Di Massimo, G. A. Montague, M. T. Tham, A. J. Morris, "Artificial neural networks in process engineering," IEE Procedings in Control Theory and Applications, vol. 138, no. 3, pp. 256-266, May 1991. 